\title{
Research to improve traction and dynamic quality of locomotives
}

\author{
Mykola Gorbunov ${ }^{1}$, Vaclav Pistek ${ }^{2}$, Maksym Kovtanets ${ }^{3}$, Olena Nozhenko ${ }^{4}$, Sergii Kara ${ }^{5}$, \\ Pavel Kučera ${ }^{6}$ \\ 1,3, 4,5Volodymyr Dahl East-Ukrainian National University, Severodonetsk, Ukraine \\ ${ }^{2,6}$ Brno University of Technology, Technicka 2896/2, 616 69, Brno, Czech Republic \\ ${ }^{1}$ Corresponding author \\ E-mail: ${ }^{1}$ gorbunov0255@gmail.com, ${ }^{2}$ pistek.v@fme.vutbr.cz, ${ }^{3}$ kovtanetsm@gmail.com, \\ 4nozhenko.olena@gmail.com, ${ }^{5}$ kara1520mm@gmail.com, ${ }^{6} \mathrm{kucera@fme.vutbr.cz}$
}

Received 3 September 2017; accepted 4 September 2017

DOI https://doi.org/10.21595/vp.2017.19043

Check for updates

\begin{abstract}
The article reviews and analyzes available theoretical and experimental studies aimed at improving the tractive and dynamic characteristics of locomotives. Based on the information received, the authors formulated the following tasks of further research to increase and stabilize traction and coupling qualities of the locomotive.
\end{abstract}

Keywords: diesel locomotives, wheel pairs, coefficient of adhesion, dynamics, traction, hanging.

\section{Introduction}

The constant growth of sectional and axial power of modern diesel locomotives with a new severity pose the problem of improving their traction qualities, improving the use of power for traction.

As shown by the conducted studies of the operating modes of the locomotive 2TE116, which is in the ordinary operation of the freight traffic, most of the time it is used at low speeds, realizing close to the maximum values of the traction force [1]. The duration of the locomotive in the range of $0-20 \mathrm{~km} / \mathrm{h}$ is $40 \%$ of the total time. For locomotives of higher power, an increase in the duration of the operation should be expected with a limited coupling power, i.e. reduce its effective use.

Therefore, the issues of increasing the maximum traction forces of locomotives and the efficiency of their use at the same time their power is constantly in the field of vision of scientists and designers.

\section{Main text}

The main indicator determining the potential for wheel-to-rail engagement is the physical coefficient of adhesion $\psi_{0}$, which is equal to the ratio of the maximum traction force $F_{a d . m a x}$ developed by a single wheel when moving off at a constant vertical load $P_{B}$ and without any dynamic disturbances to this load:

$\psi_{0}=\frac{F_{a d \cdot \max }}{P_{B}}$.

Virtually all data on the physical coefficient of adhesion were obtained experimentally. Sufficiently reliable calculation methods for its determination do not yet exist. In laboratory conditions, when steel is rubbed in steel, its values reach 0.6-0.7 [2]. Evidence of the large reserves of contact of two metal surfaces with respect to bonding are the experiments described in [3], in which it was possible to obtain values of the coefficient of friction in vacuum to 5 .

However, in practice, the physical cohesion coefficients measured on railways have a wide spread and rarely exceed $0.40-0.45$, and lower values up to 0.06 are recorded $[4,5]$. The main reason for their reduction are the contamination of the rolling surfaces of rails and wheels with oils, dust in combination with water, etc.

One of the important issues to be solved when designing the running gear of locomotives is 
the problem of uniform distribution of vertical loads on wheel pairs when the traction force is realized.

For locomotives with individual drive of wheel pairs, the maximum tractive force is limited by the binding force of the limiting axis. The maximum value of the adhesion force is defined as the sum of the traction forces of all its wheel pairs, which do not exceed the binding force of the limiting wheel:

$F_{\text {ad.max }}=n \cdot F_{\text {ad.max }}=n \cdot P_{v \min } \cdot \psi_{\max }$

where $n$ - is the number of traction axles; $F_{a d \text { max }}$ - maximum binding force of the limiting axis; $P_{v m i n}$ - vertical load on the limiting axis; $\psi_{\max }$ - the maximum coefficient of adhesion.

If the maximum traction force on the limiting wheel pair is reached, further increase of the locomotive traction force is impossible, although the adhesion forces of the other axles have not reached their limit, i.e. their traction capabilities, and as a result, the traction capabilities of the entire locomotive, are underutilized. Thus, the locomotive will realize the maximum tractive force, when the adhesion forces of all its axes are equal to each other. The reason for the appearance of the limiting wheel pair on the locomotive is, first of all, the redistribution of vertical loads due to the action of the tipping torque of the traction forces.

The dynamic nature of the realization of the traction force has a great influence on the process of coupling the locomotive with the rails. The experience of domestic and foreign locomotive construction shows that the dynamic performance of the crew is largely determined by the design and characteristics of the spring suspension. When developing perspective locomotives, designers are faced with a wide range of requirements to reduce the dynamic impact on the track, redistribute loads on wheel pairs, frame forces and angles of approach in straight and curved sections of the track, etc., which leads to the search for new technical solutions aimed at to improve the crew part in both the first and second stages of spring suspension.

The uneven distribution of vertical loads along the sides of the wheel pairs has a significant effect on the traction qualities of the diesel locomotive. According to the calculated dependencies, each percent of the difference in loads between the wheels causes an increase in the relative slip speed of the axis by $0.25-0.45 \%$, thereby increasing its propensity to boxing. The reason for the uneven distribution of loads along the sides of the wheel pair can be the errors from the weights, the instability of the locomotive body on the supports, the elevation of the rail in curves, etc. For example, when locomotives were inspected, the center of gravity of the body was shifted relative to the transverse axles of the bogies, reaching $20 \mathrm{~mm}$, which leads to a change in loads on the sides of the bogies to $20 \mathrm{kN}$ and, as a result, to an increase in the speed of relative sliding of wheel sets.

Therefore, the study of the characteristics of the connections between the body and the trolleys and the further improvement of their structures is one of the reserves to increase the traction qualities of the locomotives.

Analysis of the development of structural schemes of pedestrian suspension suspension testifies to the desire of designers to solve the problem of increasing the traction qualities of locomotives by applying a balanced spring suspension. With all the advantages of balanced suspension, it has more weight, more complicated. Moreover, this decision cannot be considered the most effective from the point of view of traction qualities, especially since the possibilities of individual suspension in this direction are far from exhausted.

In addition to the dynamical factors considered, the uneven distribution of the loads of wheel pairs is laid already at the stage of locomotive weights. When determining the traction qualities of locomotives, it is usually assumed that in the static state, all wheel pairs have the same load on the rails. The load is assumed equal to the nominal, determined by the formula:

$P_{\text {nom }}=\frac{G}{n}$ 
where $G$ - is the calculated weight of the locomotive; $n$ - number of wheelsets.

In reality, the loads from wheel sets to rails have deviations from the calculated ones, which, according to the technical specifications for the delivery of locomotives, should not exceed $\pm 3 \%$ [6]. In the mode of realization of the traction force, the most favorable conditions for the transition to boxing are created in the wheel pair that has the least load on the rails, all other conditions being equal; it is called limiting by weight. With a reduction in the actual load of the wheel pair, as compared with the rated (nominal) load by $3 \%$, a proportional decrease in its maximum tractive force occurs, i.e. the static coefficient of use of the coupling weight does not exceed 0.97 .

The reasons for the uneven distribution of loads along the wheel pairs of the locomotive in the static state may be the longitudinal displacement of its center of gravity, the difference in the rigidity of the axle springs and the body supports on the trolley, the geometric imperfections of the body frames and bogies. During the operation of locomotives, there may be a violation of the initial adjustment of axle suspension, as well as changes in the stiffness of the body supports on the trolley, for example, because of the relaxation processes occurring in the rubber elements.

The analysis of weighing results on the scales of diesel locomotives on cartilaginous carts which has individual spring suspension is given in the paper. The body weight with the equipment installed in it is transferred through eight supports with rubber-metal elements to two carts, and from them through sets of springs to wheel pairs. When weighing diesel locomotives, in case of deviation of axle loads from the required values, it is allowed to adjust the spring suspension by means of gaskets installed between the elastic elements and the supporting surfaces. Based on the results of the weighing, the statistical processing of the measurements was carried out [7]. For normal distribution conditions, the mathematical expectation, the root-mean-square deviations of the loads on each locomotive pair of diesel locomotives are determined, and the coupling weight utilization factors for weighting for medium and minimum-probable loads are calculated from the following dependencies:

$$
\begin{aligned}
& M\left(P_{s t}\right)=\frac{1}{n} \cdot \sum_{j=1}^{n} P_{j}, \\
& S=\sqrt{\frac{1}{n} \sum_{j=1}^{n}\left(P_{j}-M\left(P_{s t}\right)\right)^{2},} \\
& P_{s t}^{\text {min }}=M\left(P_{s t}\right)-2,5 \cdot S, \\
& \eta_{0}^{\text {mean }}=\frac{M\left(P_{s t}\right)}{P_{\text {nom }}}, \\
& \eta_{0}^{\text {min }}=\eta_{0}^{\text {mean }}-2,5 \cdot \frac{S}{P_{\text {nom }}} .
\end{aligned}
$$

The results of statistical processing of weightings, symbols and their decoding are presented in Table 1 and in Fig. 2.

Thus, the analysis of diesel locomotive weightings has shown that the distribution of loads on wheel pairs is characterized by the fact that the extreme wheel pairs of each trolley have loads less than nominal, and the mathematical expectations of loads of medium wheel pairs are $1-2 \%$ greater than for extreme ones. For the minimum-probable load values, this difference is $2.9-1.8 \%$, i.e. the average wheel pairs of the trolleys have some clutch reserve, in comparison with the extreme ones.

After a run of $200000 \mathrm{~km}$, the loads on the supports change even more because of the unequal height and rigidity of the rubber-metal elements. In this case, the overload of individual supports can reach 6-8 \%, which in turn leads to a change in loads on wheel pairs. Deviations of loads on wheel pairs even at not the most unfavorable combinations of deviations of loads on supports reach 4.0-4.5\% and even 6.0-7.0\%. Measurements of loads on wheel pairs in diesel locomotives 
with the above mentioned runs have shown that the difference in loads of the extreme and medium wheel sets of bogies reaches 3.0-4.5\%.

The carried out analysis of locomotive locks allows us to conclude that the average wheel pairs of each trolley are in the best clutch conditions and cannot be limiting in terms of weighting.

Table 1. The results of statistical processing of weightings, symbols and their decoding

\begin{tabular}{|c|c|c|c|c|}
\hline \multirow{2}{*}{ Index } & \multirow{2}{*}{ Symbol } & \multicolumn{3}{|c|}{ Wheel pair number } \\
\cline { 3 - 5 } & & $1(6)$ & $2(5)$ & $3(4)$ \\
\hline Number of experiments & $n$ & 200 & 200 & 200 \\
\hline $\begin{array}{c}\text { Mathematical expectation of the load } \\
\text { from the wheel pair on the rails, } \mathrm{kN}\end{array}$ & $M\left(P_{s t}\right)$ & 226.1 & 230.49 & 228.1 \\
\hline Root-mean-square deviation of load, $\mathrm{kN}$ & $S$ & 2.77 & 1.67 & 2.6 \\
\hline Minimum-probable load value, $\mathrm{kN}$ & $P_{s t}^{\text {min }}$ & 219.1 & 225.8 & 221.6 \\
\hline $\begin{array}{c}\text { Coefficient of use of coupling weight } \\
\text { for mathematical expectation of loading }\end{array}$ & $\eta_{0}^{\text {mean }}$ & 0.983 & 1.002 & 0.991 \\
\hline $\begin{array}{c}\text { Coefficient of use of coupling weight } \\
\text { for the minimum-probable load value }\end{array}$ & $\eta_{0}^{\text {min }}$ & 0.952 & 0.981 & 0.963 \\
\hline
\end{tabular}

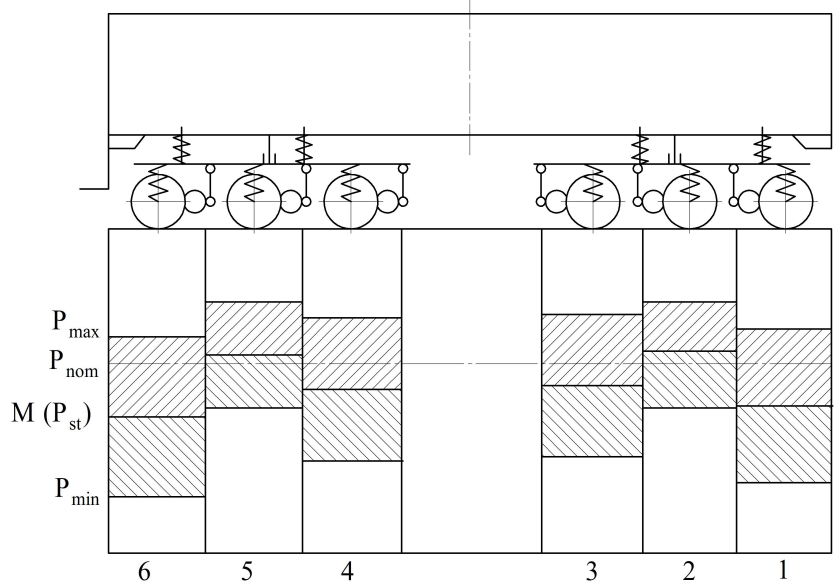

Fig. 2. Static characteristics of load distribution from wheel pairs to rails

The maximum traction force of a locomotive wheel pair can be represented as a function of two variables - the component of the vertical load per axle $P_{v i}$ and the coefficient of adhesion $\psi_{\text {maxi }}$. The task of realizing the maximum traction force $F_{a d \text { max }}^{l o k}$ by a locomotive with an individual drive of wheel sets will obviously be solved if all wheel pairs reach their limits of adhesion at the same time:

$F_{a d . \max 1}=F_{a d \cdot \max 2}=\ldots \cdot F_{a d . \max n}, \quad$ or $P_{v i} \cdot \psi_{\max i}=i d e m, \quad(i=1,2 \ldots, n)$.

One of the main ways to solve this problem is the creation of devices and structures of the running parts that allow to equalize the static components of the load from the wheel sets to the rails by compensating for their redistribution in the traction mode. There is a whole direction to increase the coefficient of use of the coupling weight, which, in fact, characterizes the degree of unevenness of these loads.

Experimental and theoretical studies noted that the coefficients of vertical dynamics for the first wheel pair, as a rule, 40-60 \% higher than the second, and 15-20\% higher than the third. The coefficients of horizontal dynamics for the first wheel pair are accordingly higher by $40 \ldots 50 \%$ and by $20-25 \%$. Cleaning the rails ahead of the wheel pair increases the physical coefficient of adhesion in the second and third wheel pairs, respectively, by $2-6 \%$ and 6-12\%. 
Coefficient of use of adhesion is an integral indicator characterizing the reduction of the maximum coefficient of adhesion in comparison with the physical from various factors. We represent the coefficient of utilization of the cohesion of the $i$ th wheel pair in the form of a product:

$\chi_{i}=\Pi_{j=1}^{N} \cdot \chi_{i j}$

where $\chi_{i j}$ - coefficient of use of adhesion from the $j$ th factor; $N$ - the number of factors considered.

Information on the magnitude of the coefficients $\chi_{i j}$ is extremely limited. The definition of their specific values is complicated by the dependence on the design of the crew part of locomotives.

This approach allowed us to propose a method for increasing the traction qualities of a locomotive, consisting in deliberately changing the loads from wheel sets to rails in order to maximize the closest traction forces of wheel sets. As an objective function in the solution of the problem can be adopted:

$C=\sum_{j=1}^{n} \cdot \sum_{j=1}^{n}\left|P_{v i} \cdot \psi_{\text {maxi }}-P_{v v} \cdot \psi_{\text {maxvi }}\right| \rightarrow \min$,
$C=\sum_{j=1}^{n} \cdot \sum_{j=1}^{n}\left|P_{v i} \cdot \chi_{i j}-P_{v v} \cdot \chi_{v j}\right| \rightarrow \min$.

In this case, the coefficients of the use of cohesion $\chi_{i j}$ with its complex structure are considered as quantities with limited control capabilities, while a change in the value $P_{v i}$ allows the minimum of the objective function to be achieved most simply and effectively.

\section{Conclusions}

Thus, based on the review and analysis, available theoretical and experimental studies aimed at improving the tractive and dynamic characteristics of locomotives, the following research problems are formulated $[8,9]$ :

- development of a method for increasing the traction qualities of a locomotive on the basis of accounting for the degree of change in the coupling capabilities of each wheel pair of the crew under the influence of various factors and its implementation in the construction of the locomotive by deliberately changing the loads of wheel sets on rails to equalize the maximum traction forces on all wheel pairs;

- obtaining quantitative characteristics of the influence of dynamic factors on the traction capabilities of each wheel pair and locomotive in general;

- investigate the influence of the first and second stages of spring suspension on the dynamic and traction qualities of the locomotive;

- creating a study of technical solutions to improve the dynamic and traction qualities of the locomotive;

- the creation of bench equipment and measuring devices for the experimental study of the characteristics of experimental units and there can be used other methods of the measurement $[10,11]$.

\section{Acknowledgements}

The research was held on the basis of the scientific research "Creating a multi-functional high-end energy management engineering contact surfaces "wheel-rail" for eco-efficient power transmission", funded by the Ministry of Education and Science of Ukraine. The publication 
contains the results of studies conducted by President's of Ukraine grant for competitive Projects F70 "Creation of multifunctional scientific methods and tools for combined management of wheel-brake-rail system for prevention of accidents, elimination of the environmental disasters risk" of the State Fund for Fundamental Research. This work is also an output of the internal BUT research Project Reg. No. FSI-S-17-4104.

\section{References}

[1] Kudinov V. S., Dolgopolov S. I. Modes of operation of locomotives 2TE116. Transport Engineering, 1979, p. 13-15.

[2] Genkin M. D., Misharin Yu. A., Prokhorov V. S. Investigation of the nature of the change in friction coefficients for rolling with low slip. Mechanical Engineering, 1960, p. 63-68.

[3] Novion M., Bernard M. Connaissancer Nokvelles sur Ladherenee des Locomotives Electrigues, Revu Jenerala dos Chemins de fer. 1961, p. 133-157.

[4] Luzhnov Ju. M. Clutch Wheel Rails (Nature and Regularity). Moscow, Intekst Publ., 2003, p. 144.

[5] Popov V. A. Influence of Frictional Processes on the Realization of the Adhesion of Locomotive Wheels to Rails. Ph.D. Thesis, Moscow, 1984, p. 206.

[6] Fufryansky N. A., Nesterov A. S., Dolganov A. N. Development of Locomotive Traction. Moscow, Transport, 1982, p. 303.

[7] Gorbunov N. I., Slashchev V. A., Kryshtal N. I. Features of hanging locomotives of type 2TEP6, 2TE10M and ways to improve their traction qualities. Voroshilovgrad, 1986, p. 11.

[8] Gorbunov N. I. Increase of Traction Qualities of Diesel Locomotives at the Expense of Improvement of Elastic Ties of Bogies. Ph.D. Thesis, Dnipropetrovsk, 1987, p. 180.

[9] Kovtanets M. V. Improving the Cohesion Characteristics of the Locomotive with the Jet-Abrasive Impact on the Contact Zone of the Driving Wheel and Rail. Ph.D. Thesis, Severodonetsk, 2015, p. 206.

[10] Tuma J. Vehicle Gearbox Noise and Vibration: Measurement, Signal Analysis, Signal Processing and Noise Reduction Measures. John Wiley, Chichester, 2014.

[11] Porteš P., Kučera P., Píštěk V., Fojtášek, J., Zháňal L. Modern tools for vehicle development. Engineering Mechanics, 2017, p. 54-57. 